\title{
In(Ga)As self-assembled quantum ring formation by molecular beam epitaxy
}

\author{
Daniel Granados and Jorge M García ${ }^{a)}$ \\ Instituto de Microelectrónica de Madrid, CNM, CSIC, Isaac Newton, 8, Tres Cantos, 28760-Madrid, Spain
}

(Received 9 September 2002; accepted 14 February 2003)

\begin{abstract}
The effect of growth conditions on the morphological properties of InAs/GaAs(001) quantum dots covered by a thin $(<3 \mathrm{~nm}) \mathrm{GaAs}$ cap has been studied by atomic force microscopy. Each dot turns into an elongated nanostructure at $540{ }^{\circ} \mathrm{C}$ upon deposition of the cap in $\mathrm{As}_{4}$ atmosphere, while structures with two humps are obtained when capping at $500^{\circ} \mathrm{C}$. The use of $\mathrm{As}_{2}$ atmosphere instead of $\mathrm{As}_{4}$ at $500{ }^{\circ} \mathrm{C}$ leads to the formation of quantum rings. Photoluminescence spectroscopy and polarization photoluminescence (PL) at $15 \mathrm{~K}$ show dramatic changes due to the different kinds of confinement. This allows the possibility of tailoring PL emission by controlling the size and shape.

(C) 2003 American Institute of Physics. [DOI: 10.1063/1.1566799]
\end{abstract}

There is increased interest nowadays in methods to control the size and shape of quantum dots (QDs) to tune QD optical properties. In particular, morphological changes of QD grown by molecular beam epitaxy (MBE) due to a thin GaAs cap have been reported. ${ }^{1}$ This approach has turned into a powerful technique by which to obtain self-assembled nanostructures such as quantum rings (QRs). ${ }^{2-4}$ Although the first QRs were grown some years ago, only a few groups have reported similar findings. In some cases ${ }^{4,5}$ metalorganic precursors were used, and in others in which MBE was used, ${ }^{6}$ the ring-like structures were related to InAs "giant" QDs, probably associated with dislocated islands.

In this work we present results of QDs covered with a 2 $\mathrm{nm}$ GaAs cap layer. We show that the final morphology and optical properties depend strongly on the starting shape of the QD and on detailed growth conditions such as the substrate temperature for the capping process $\left(T_{\text {cap }}\right)$ and the molecular species of the As flux.

The final sample morphology was characterized by ex situ contact mode atomic force microscopy (AFM). Their emission characteristics are measured by photoluminescence (PL) spectroscopy and polarization photoluminescence (PPL) with a $514.5 \mathrm{~nm}$ Ar laser line and a $0.22 \mathrm{~m}$ monochromator with a liquid nitrogen cooled Ge diode. For PPL measurements the $\langle 110\rangle$ direction of the sample is oriented $45^{\circ}$ with respect to the direction of laser polarization. A GlanThomson polarizer is placed at the entrance of the monochromator and is used to measure PL intensity along $\langle 110\rangle$ and $\langle 1 \overline{1} 0\rangle$ directions $\left(\mathrm{PL}_{110}, \mathrm{PL}_{1 \mathrm{I} 0}\right)$. The polarization degree (PD) is defined as $\mathrm{PD}=\left(\mathrm{PL}_{1 \mathrm{TO}}-\mathrm{PL}_{110}\right) /\left(\mathrm{PL}_{1 \mathrm{~T} 0}+\mathrm{PL}_{110}\right)$.

The substrate temperature $(T)$ is carefully calibrated by observation of the reflection high energy electron diffraction (RHEED) diffraction pattern, the oxide desorption $\left(\sim 630^{\circ} \mathrm{C}\right)$ and surface reconstruction phase transition from $c(4 \times 4)$ to a clear $2 \times 4$. This transition occurs at $T=520^{\circ} \mathrm{C}$ and at an $\mathrm{As}_{4}$ beam equivalent pressure (BEP) of 3.5 $\times 10^{-6}$ mbar $30 \mathrm{~s}$ after shutting down the As source, and is observed by RHEED. The QDs are grown by depositing 1.7

${ }^{a)}$ Electronic mail: jorgem@imm.cnm.csic.es monolayers (MLs) of InAs at $540^{\circ} \mathrm{C}$, using $\sim 3-4 \times 10^{-6}$ mbar BEP-As 2 pressure (a incipient $2 \times 4$ RHEED pattern reconstruction is observed). InAs deposition takes place in a growth sequence of $0.1 \mathrm{ML}$ InAs (at $\sim 0.06 \mathrm{ML} / \mathrm{s}$ ) plus a $2 \mathrm{~s}$ pause under As flux. The QDs are next annealed $1 \mathrm{~min}$ to enhance the size distribution and to obtain low density ensembles $\left(10^{8}-10^{9} \mathrm{~cm}^{-2}\right)$, which is required for improved AFM analysis. Subsequently, a GaAs thin cap layer is grown (1 ML/s) at $T_{\text {cap }}$ and annealed for $1 \mathrm{~min}$. The samples for AFM characterization are cooled down immediately and removed from the chamber. The samples for PL measurements are obtained by burying these transformed nanostructures under a thick GaAs layer where the first $20 \mathrm{~nm}$ of GaAs is grown at $T_{\text {cap }}$ and then $T$ is increased to $595^{\circ} \mathrm{C}$.

Figure 1(a) shows an AFM image of QDs with a full width at half maximum (FWHM) vertical size distribution of $9 \%$ centered at $\sim 10 \mathrm{~nm}$. When a $2 \mathrm{~nm}$ cap is deposited at $540^{\circ} \mathrm{C}$ under $4-5 \times 10^{-6} \mathrm{mbar}^{\mathrm{As}} \mathrm{s}_{4} \mathrm{BEP}$ each QD is trans-
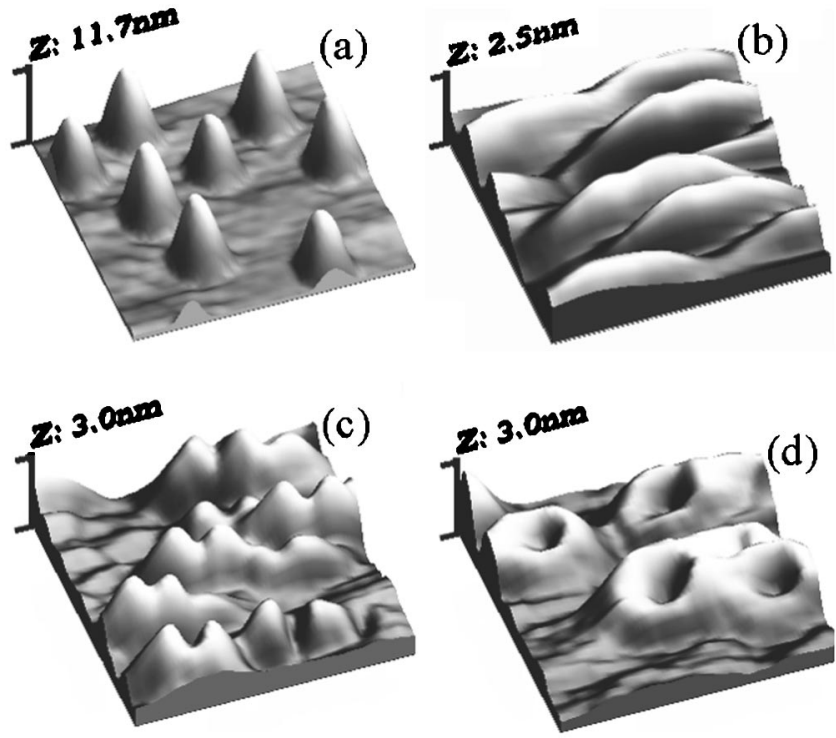

FIG. 1. $250 \mathrm{~nm} \times 250 \mathrm{~nm}$ AFM images of (a) a QD grown at $540{ }^{\circ} \mathrm{C}$, (b) a QD after $2 \mathrm{~nm}$ capping at $540{ }^{\circ} \mathrm{C}$, (c) a QD after $2 \mathrm{~nm}$ capping at $500{ }^{\circ} \mathrm{C}$ and (d) a QD after $2 \mathrm{~nm}$ capping at $500{ }^{\circ} \mathrm{C}$ with $\mathrm{As}_{2}$. 
formed into an elongated dash-like nanostructure (160 $\mathrm{nm} \times 40 \mathrm{~nm}) \sim 2 \mathrm{~nm}$ high [Fig. 1(b)]. Cap deposition at lower temperatures, $T_{\text {cap }}=500^{\circ} \mathrm{C}$, produces two camel hump-like nanostructures $(100 \mathrm{~nm} \times 50 \mathrm{~nm}) \sim 2.5 \mathrm{~nm}$ high [Fig. 1(c)]. Figure 1(d) shows quantum rings $(100 \mathrm{~nm} \times 90 \mathrm{~nm}) \sim 1.5 \mathrm{~nm}$ high, which formed at $T_{\text {cap }}=500{ }^{\circ} \mathrm{C}$ under $\mathrm{As}_{2}$ flux. It is worth mentioning that we never obtained QRs for samples with initially low $(<7 \mathrm{~nm})$ QD height.

These morphological changes allow a better understanding of the quantum ring formation mechanism. Some authors have suggested ${ }^{4,7,8}$ that a change in the balance of surface free energy due to the thin capping layer creates an outward pointing force. This force brings about material redistribution and results in a ring shaped structure. Although this would imply a liquid InAs QD, there are recent experimental ${ }^{9}$ and theoretical results ${ }^{10}$ showing that biaxial epitaxial stress of InAs on $\mathrm{GaAs}(001)$ leads to a mixture of stress-free matter including phases of "floating" liquid In and InAs. Moreover, this effect has been observed ${ }^{11}$ independent of $T$ in the 170$520^{\circ} \mathrm{C}$ range. The driving force for this melting is the elastic energy, ${ }^{9}$ it is expected that when a relaxed QD is partially covered, and consequently compressed, it undergoes a phase transition into liquid phase, and allows a de-wetting process ${ }^{7}$ that expels the InAs from the QD. We have to consider also the simultaneous $\mathrm{Ga}$-In alloying process that takes place. The presence of liquid InAs is basically a $T$ independent process ${ }^{11}$ whereas Ga-In alloying is strongly $T$ dependent. ${ }^{12}$ The competition between these two processes at different $T_{\text {cap }}$ can explain our experimental observations. Although liquid InAs must have very high mobility, at large temperatures $\left(T_{\text {cap }}=540^{\circ} \mathrm{C}\right)$ the strong In-Ga alloying processes result in the formation of an immobile ${ }^{13}$ InGaAs phase at the base of the dot, resulting in a dash-like structure [Fig. 1(b)]. For lower cap temperatures $\left(T_{\text {cap }}=500{ }^{\circ} \mathrm{C}\right)$, the migration of $\mathrm{Ga}$ atoms into the center region is reduced and dewetting of the liquid center InAs region leads to the formation of a depleted region surrounded by immobile InGaAs. This causes the camel hump-like shape structures.

The observation of quantum ring formation under $\mathrm{As}_{2}$ is related to enhancement of the reactivity along the $\langle 110\rangle$ direction and it is discussed next. During GaAs homoepitaxy, it is well known that the migration distance of both principal directions is different due to the anisotropic chemical reactivity of the steps. ${ }^{14}$ Arsenic terminated $B$ steps (perpendicular to the $\langle 1 \overline{1} 0\rangle$ direction) provide highly active sites for gallium adsorption whereas gallium terminated $A$ steps are less reactive to group III migrating atoms. The structures elongated along the $\langle 1 \overline{1} 0\rangle$ direction usually found when growing by MBE and step flow growth mode in $B$ type vicinal surfaces are attributed to this chemical reactivity anisotropy. The step flow of $A$-type facets can be achieved for higher As pressures. ${ }^{14}$ If we assume similar chemical behavior of the original InAs island steps, an increase of As pressure will produce enhancement of the reactivity of $A$ steps to $\mathrm{Ga}$ atoms. The use of $\mathrm{As}_{2}$ then has similar effects to employing a larger $\mathrm{As}_{4}$ flux. ${ }^{15}$

Figure 2 (squares) shows PL emission from QDs at 15 $\mathrm{K}$. The interband transitions between the ground and excited first states of electrons and holes are observed at low power excitation $\left(\sim 20 \mathrm{~W} \mathrm{~cm}^{-2}\right)$. The PL spectrum of dash-like Downloaded 14 May 2010 to 161.111 .235 .252 . Redistribution subje

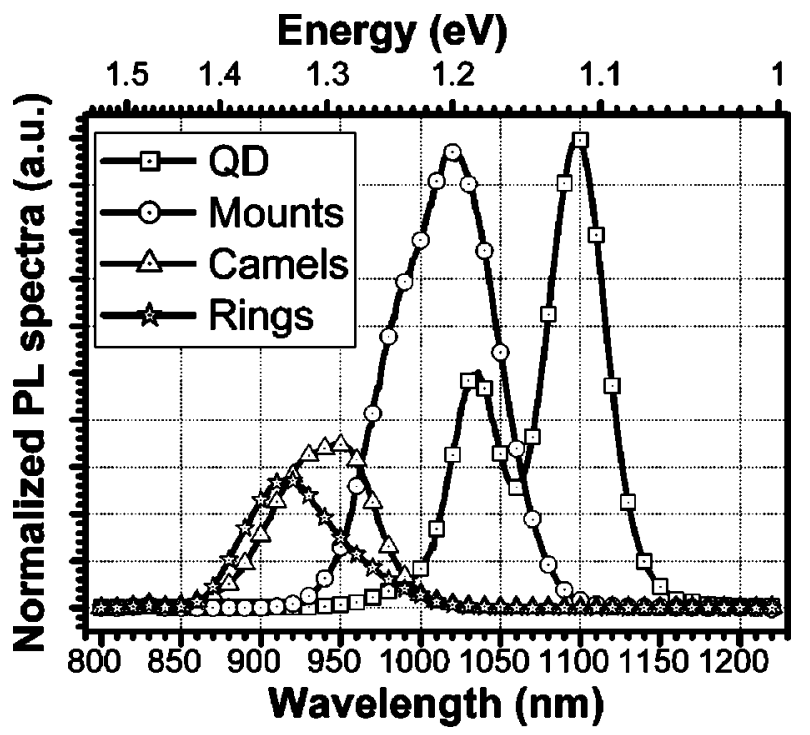

FIG. 2. Low temperature PL spectra at $20 \mathrm{~W} / \mathrm{cm}^{2}$ of samples with the nanostructures shown in Fig. 1 buried with GaAs. Strong blueshifting of the wavelength is observed from the dot to ring emission.

nanostructures (Fig. 2, circles) is blueshifted $\sim 85 \mathrm{meV}$ with respect to the QD emission, in agreement with the AFM measured reduction in vertical size of the original QDs. Although the camel hump-like nanostructures are slightly taller $(0.5 \mathrm{~nm})$ than the dash-like ones, the PL emission (Fig. 2, triangles) shows a larger blueshift than that for the former ones (178 meV). The PL emission of the QR (Fig. 2, stars) shows the largest blueshift $(220 \mathrm{meV})$ with respect to the dots. This larger blueshift for camel hump-like and QR nanostructures suggests a reduction of confinement, probably associated with the formation of the center depleted region.

The polarization degree for large pumping power of a QD sample is 9\% as shown in Fig. 3(a). Similar spectra have been observed by Noda et al. ${ }^{16}$ who also showed theoretically that the polarization properties of the transitions of slightly asymmetrical QDs can be assigned to transitions between excited states of electrons and holes with the same quantum number. The PPL signal is positive (stronger PL along $\langle 1 \overline{1} 0\rangle$ ) due to overlapping of the emission modes and the predominance of lower energy states due to band-filling effects. ${ }^{16}$ For the QR sample, and in spite of the more asymmetrical shape of the rings [Fig. 3(b)], its PPL spectrum is $6 \%$, somewhat smaller than the dot polarization degree. From 950 to $1025 \mathrm{~nm}$ the PL signal along $\langle 110\rangle$ is more intense than along $\langle 1 \overline{1} 0\rangle$. Accordingly the polarization degree exhibits a negative character in this wavelength of emission, contrary to what is observed in the QD measurements. This suggests different spatial distributions of the wave function envelopes. The PPL signal of camel hump-like and dashed structures shows no clear trend and is currently under investigation. It has been recently shown ${ }^{17}$ that a giant vertical permanent dipole moment opposite in sign to the observed one in QDs is present in similar quantum ring nanostructures. This change in sign may be associated with the distribution of InAs vertically inverted to the one in the QD. Whereas QDs have a high $\operatorname{In}(\mathrm{Ga})$ As content at the base, the quantum rings seem to present a high $\operatorname{In}(\mathrm{Ga})$ As content on the top and around the crater.

to AIP license or copyright; see http://apl.aip.org/apl/copyright.jsp 


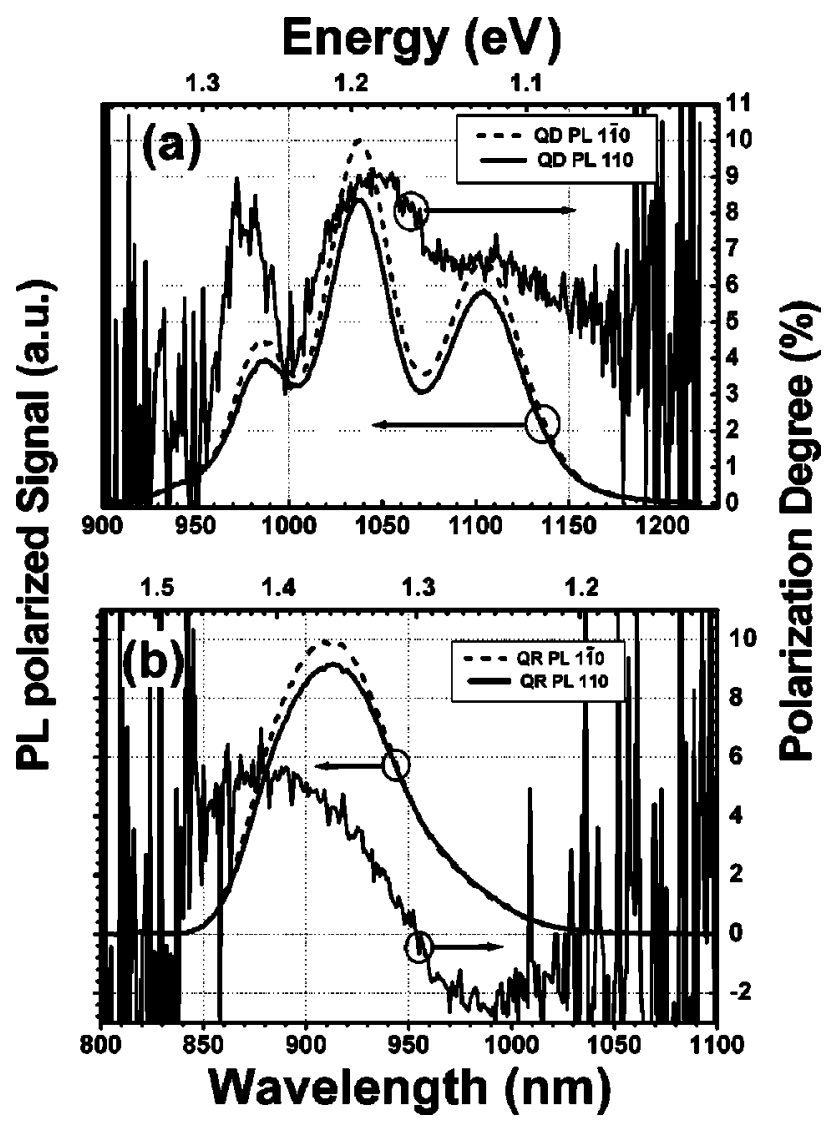

FIG. 3. Polarization PL emission at $20 \mathrm{~K}$ using $1000 \mathrm{~W} / \mathrm{cm}^{2}$ of a sample with (a) buried dots and (b) buried rings.

Several conclusions as to the transformation mechanisms involved during self-assembled nanostructure thin GaAs capping can be suggested. The InAs within the QD is destabilized by the deposition of GaAs, thus driving material redistribution. A high migration rate of liquid phases In and InAs enhances mass transport out toward the center region of the $\mathrm{QD}$, while $\mathrm{Ga}$-In alloying effects reduce the mobility and tend to prevent the formation of a crater at high substrate temperatures. Quantum ring formation is achieved at low substrate temperatures. The use of $\mathrm{As}_{2}$ is required for successful isotropic distribution of the InGaAs forming the rim. Large QDs of more than $7 \mathrm{~nm}$ in height are necessary to obtain a depleted center region. Understanding the mechanisms by which these nanostructures grow and change shape allows tailoring of their optical properties such as PL wavelength emission and polarization response.

The authors would like to thank J. L. Costa Kramer for help during writing and Axel Lorke and Richard Warburton for fruitful discussions. This work was partially supported by Project No. 07T/0062/2000 of the Comunidad Autónoma de Madrid, Project No. TIC99-1035-C02, Nanoself Project No. TIC2002-04096-C03 and by Nanomat of the EC Growth Program, Contract no. G5RD-CT-2001-00545.

${ }^{1}$ J. M. García, G. Medeiros-Ribeiro, K. Schmidt, T. Ngo, and P. M. Petroff, Appl. Phys. Lett. 71, 2014 (1997); R. Songmuang, S. Kiravittaya, and O. G. Schmidt, J. Cryst. Growth 249, 416 (2003).

${ }^{2}$ A. Lorke, R. J. Luyken, A. O. Govorov, J. Kotthaus, J. M. García, and P. M. Petroff, Phys. Rev. Lett. 84, 2223 (2000).

${ }^{3}$ R. J. Warburton, C. Schaflein, D. Haft, F. Bickel, A. Lorke, K. Karrai, J. M. García, W. Schoenfeld, and P. M. Petroff, Nature (London) 405, 926 (2000).

${ }^{4}$ T. Raz, D. Ritter, and G. Bahir, Appl. Phys. Lett. 82, 1706 (2003).

${ }^{5}$ J. S. Lee, H. W. Ren, S. Sugou, and Y. Masumoto, J. Appl. Phys. 84, 6686 (1998).

${ }^{6}$ I. Kamiya, I. Tanaka, and I. Sakaki, J. Cryst. Growth 201, 1146 (1999).

${ }^{7}$ A. Lorke, R. Blossey, J. M. García, M. Bichler, and G. Abstreiter, Mater. Sci. Eng., B 88, 225 (2002).

${ }^{8}$ R. Blossey and A. Lorke, Phys. Rev. E 65, 021603 (2002).

${ }^{9}$ J. M. García, J. Silveira, and F. Briones, Appl. Phys. Lett. 77, 409 (2000).

${ }^{10}$ D. Bottomley, Appl. Phys. Lett. 80, 4747 (2002).

${ }^{11}$ J. Silveira, J. M. García, and F. Briones, J. Cryst. Growth 227-228, 995 (2001).

${ }^{12}$ P. B. Joyce, T. J. Krzyzewski, G. R. Bell, B. A. Joyce, and T. S. Jones, Phys. Rev. B 58, R15981 (1998).

${ }^{13}$ A. Lorke, R. J. Luyken, J. M. García, and P. M. Petroff, Jpn. J. Appl. Phys., Part 1 40, 1857 (2001).

${ }^{14}$ Y. Horikoshi, H. Yamaguchi, F. Briones, and M. Kawashima, J. Cryst. Growth 105, 326 (1990).

${ }^{15}$ T. Ogura, D. Kishimoto, and T. Nishinaga, J. Cryst. Growth 226, 179 (2001).

${ }^{16}$ S. Noda, T. Abe, and M. Tamura, Phys. Lett. B 58, 7181 (1998).

${ }^{17}$ R. J. Warburton, C. Schulhauser, D. Haft, C. Schaflein, K. Karrai, J. M. García, W. Schoenfeld, and P. M. Petroff, Phys. Rev. B 65, 113303 (2002). 